\title{
Factores clave del Teletrabajo que inciden en la relación ganar-ganar entre el trabajador y la empresa costarricense
}

\section{Key Tele-work factors that affect the win-win relation between the worker and the Costa Rican company}

MBA. Gabriel Silva Atencio

Tecnológico de Costa Rica, Costa Rica

gabriel_silva@yahoo.com
Dr. Federico Li Bonilla

Universidad Estatal a Distancia, Costa Rica

fli@uned.ac.cr
Fecha de recepción: 24 de octubre de 2019

\section{RESUMEN}

El teletrabajo podría conver tirse en una alternativa que permita al sector empresarial y al trabajador obtener una relación ganar-ganar para salir adelante ante la situación global de las empresas. El objetivo de este artículo es identificar los factores que permiten, desde el enfoque empresarial, que el trabajador pueda ejecutar actividades laborales acorde a las expectativas de la empresa y alcanzar las metas planteadas como parte del puesto de trabajo. La información fue recopilada por medio de entrevistas en profundidad y encuestas a empresas que realizan de forma exitosa esta modalidad y que han alcanzado las metas planteadas como organizaciones.

\begin{abstract}
ABSTRAC
Telework could become an alternative that may enable the business sector and the worker to obtain a win-win relation to move on in view of the global situation of the enterprises. This article aims at identifying the factors that may enable the worker, from the perspective of the business sector, to carry out activities according to the expectations of the enterprise and reach the goals established as part of the job post. The information was gathered by means of in-depth interviews and surveys to enterprises that have implemented this modality with success and have reached the goals established as organizations.
\end{abstract}

Fecha de aceptación: 22 de abril de 2020

\section{RÉSUMÉ}

Le télétravail pourrait se convertir en une alternative qui permette au monde des affaires et au travailleur d'obtenir une relation gagnant-gagnant pour aller de l'avant en fonction de la situation globale des entreprises. L'objectif de cet article est d'identifier les facteurs qui, sous l'angle des entreprises, conduisent le travailleur à pouvoir réaliser ses activités professionnelles en fonction des expectatives de l'entreprise et atteindre les objectifs fixés comme partie intégrante du poste de travail. L'information a été recueillie au moyen d'entrevues en profondeur et de sondages dans des entreprises qui réussissent à effectuer cette modalité et sont parvenues aux objectifs déterminés en tant qu'organisations.

\section{RESUMO:}

O teletrabalho poderia se tornar uma alternativa que permita ao setor empresarial e ao trabalhador obter um relacionamento de ganhar - ganhar para sair avante perante a situação global das empresas. O objetivo deste artigo é identificar os fatores que permitem, desde a perspectiva empresarial, que o trabalhador possa executar atividades trabalhistas de acordo com as expectativas da empresa e atingir as metas estabelecidas como parte da função do trabalho. As informações foram coletadas por meio de entrevistas e enquetes aprofundadas a empresas que realizam com sucesso essa modalidade e que têm atingido as metas estabelecidas como organizações.

\section{PALABRAS CLAVE: \\ COMPETENCIAS LABORALES, COMPETITIVIDAD, INNOVACIÓN, PRODUCTIVIDAD Y TELETRABAJO.}

KEY WORDS:

JOB SKILLS, COMPETITIVENESS,

INNOVATION, PRODUCTIVITY AND TELEWORK.
PALVRAS CHAVE :

COMPETÊNCIAS

TRABALHISTAS,

COMPETITIVIDADE,

INOVAÇÃO,

PRODUTIVIDADE E

TELETRABALHO.
MOTS CLÉS:

COMPÉTENCES PROFESSIONNELLES, COMPÉTITIVITÉ, INNOVATION, PRODUCTIVIÉ ET TÉLÉTRAVAIL. 


\section{INTRODUCCIÓN}

A lo largo de la historia, el ser humano ha contribuido con el mundo que hoy conocemos, por medio de los procesos de modernización evidenciados en la historia universal contemporánea; desde la primera revolución industrial con la aparición de la máquina de vapor, seguidos por el motor de combustión interna y por último la revolución de la información e inteligencia artificial, que se caracterizó por la aparición de las computadoras y las redes de comunicación y que evolucionaria hacia el Internet, llegando en pocos años con la ayuda de las nuevas tecnologías desarrolladas a dar inicio a la sociedad de la información y del conocimiento (Drucker, 1993).

El inicio del siglo XXI, ha estado enmarcado por la incorporación en la vida cotidiana y profesional de los trabajadores a la era de la información, la influencia de las tecnologías de información y comunicación, han causado una rotura en el paradigma en las relaciones laborales entre los empleados y las organizaciones, en donde el modelo de teletrabajo, surge como una posible respuesta ante este fenómeno laboral. Hodson (1994) como parte del Instituto de Economía Alemana (IWD) difundió un breve informe, donde muestra datos del estudio de SW2000 realizado por la OCDE, el cual indica: "el teletrabajo, según expertos llegará a convertirse en la nueva modalidad del siglo XXI, se está abriendo paso en importantes sectores de la economía relacionados fundamentalmente con los servicios. La rápida expansión de las tecnologías de la información hace prever que en el futuro aumente considerablemente el número de personas que realicen su actividad sin necesidad de acudir a un lugar fijo de trabajo y que estarán conectadas con sus empresas a través de un ordenador personal, el correo electrónico o Internet" (Hodson, 1994). Adicionalmente, la influencia que ejercen la innovación y evolución de las tecnologías en la vida profesional y personal de los individuos, han permitido una rápida asimilación dentro de ciertos ámbitos de la sociedad.

El propósito del presente artículo comprende la identificación de las capacidades necesarias para el teletrabajo empresarial, que contribuyen con cada uno de los vértices de la estrategia organizacional para alcanzar las metas de productividad y competitividad de las empresas, por lo cual se plantea como objetivo la identificación de los factores clave que inciden en la relación ganar-ganar entre el trabajador y la empresa.

\section{Revisión de la literatura}

En el siglo XXI, las TIC se han convertido en una herramienta crítica para el desarrollo de las actividades laborales de los individuos, convirtiéndose en una pieza vital en las sociedades de información o conocimiento. Adicionalmente, el ser humano moderno requiere de acceso a la información de forma rápida, oportuna, explicita, pertinente y fácil. Castells (1997), mencionaba que dentro de las TIC se existe: todo lo relacionado con microelectrónica, informática (máquinas y software), las telecomunicaciones (televisión y radio), que permiten al usuario el poder procesar la información de forma correcta para transformarla en conocimiento. Por lo cual, el comprender los diferentes eventos que han acontecido a lo largo de la evolución tecnológica es de vital importancia para entender la situación actual en la que se encuentra la humanidad en la era digital.

La evolución del computador personal, se ha encontrado enmarcada por una serie de eventos históricos desde el siglo pasado. En la Figura 1, se muestra una escala de tiempo con los principales eventos que señalaron la evolución del computador personal, desde el año 1935 hasta nuestros días. 
Figura 1. Cronología de la historia del computador personal

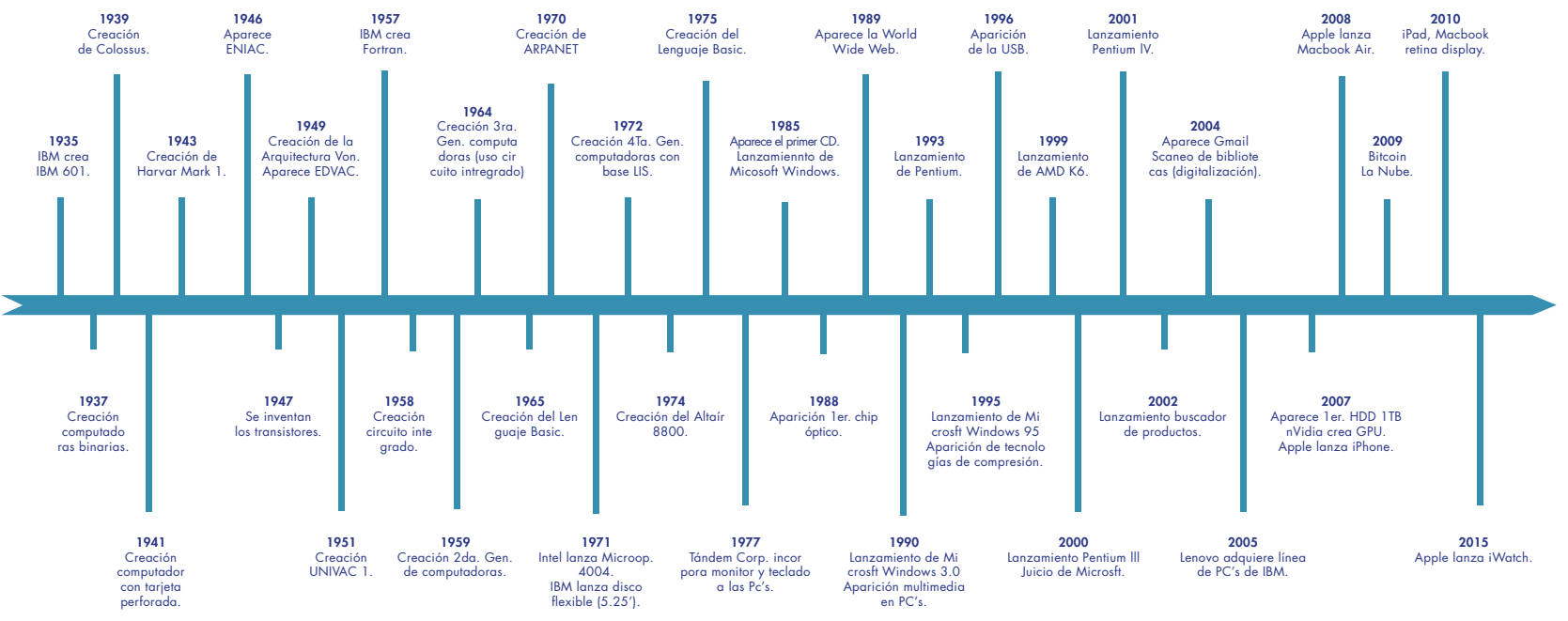

Fuente: Computer History Museum: Timeline of Computer History (2016).

La evolución del computador personal se ha caracterizado por la miniaturización de los equipos, el incremento de la velocidad de procesamiento y el aumento en la capacidad de almacenamiento. Adicionalmente, el Internet ha cambiado el paradigma del individuo en el desarrollo de su vida cotidiana y forma de laborar dentro de la sociedad, convirtiéndose en una pieza clave en la era de la información.

En la Figura 2, se muestra la evolución histórica del fenómeno de Internet desde su aparición en el año de 1962 hasta la actualidad.

Figura 2. Evolución del Internet desde 1962 hasta 2015

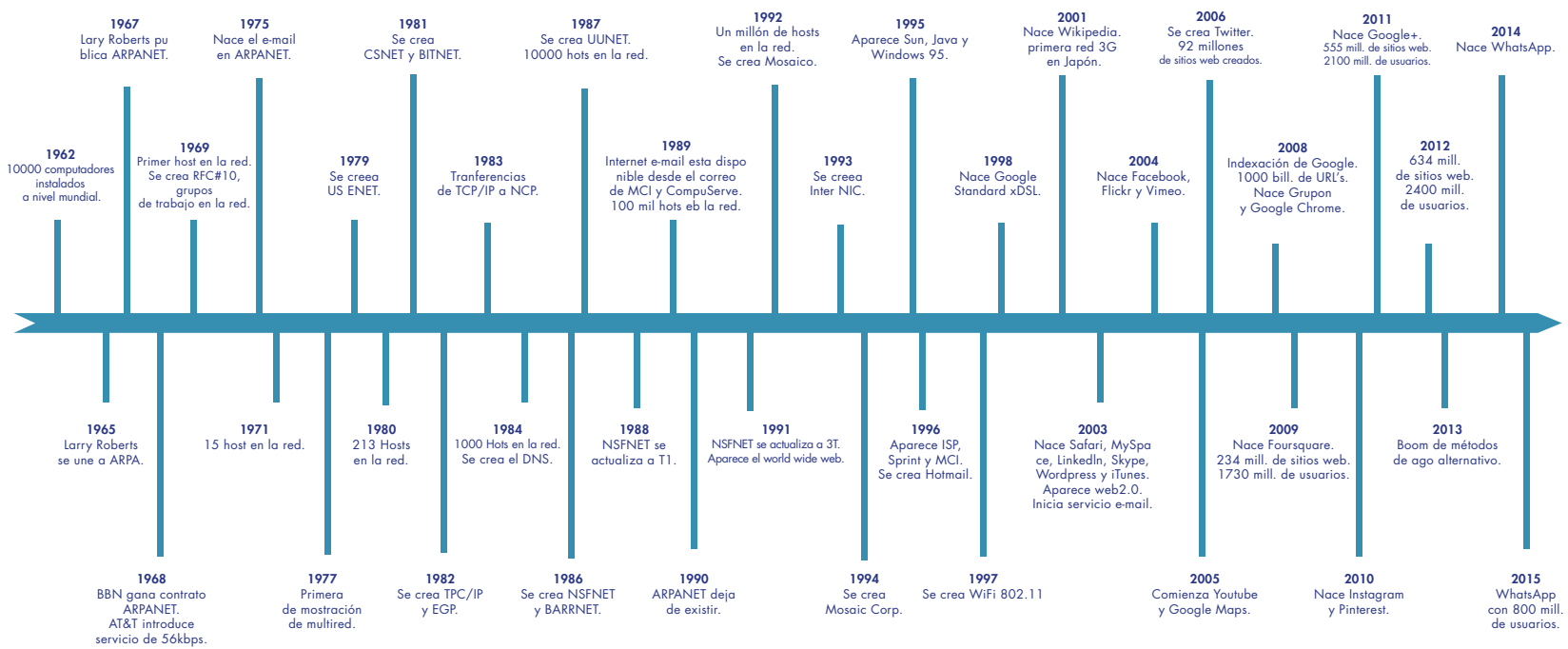

Fuente: Computer History Museum: Internet History (2016).

A lo largo de la historia el comportamiento de la tecnología y cibernética ha contribuido con la competitividad de las empresas, en modalidades laborales como el teletrabajo, generando ventajas competitivas en su entorno, sector o industria, gracias a la reducción en los precios de adquisición, la transición de las actividades laborables de entornos físicos a entornos virtuales y la contribución de las TIC en los factores de producción. 


\section{El impacto en el precio del computador personal}

En la academia Evan, Michael y Stebbins (2016) hicieron mención acerca de la llegada de las computadoras personales, las cuales son consideradas uno de los desarrollos tecnológicos más importante del siglo XX. En los años 70, llegaron a ser accesible al público general, debido a que la tecnología evolucionó al mejorar su tamaño, llegando a crear equipos más compactos. Los ordenadores personales se caracterizaban en esta época por su innovación en tamaño y costo. En la Figura 3 se aprecia que al evolucionar la tecnología los precios se reducen por gigabyte de almacenaje en función del tiempo, lo cual permite que sea más accesible al presupuesto accesible al comprador. Aunque, los ordenadores personales en los años setenta, eran más grandes y costosos de lo que son hoy, presentaban una mejora significativa sobre otras máquinas disponibles en ese entonces.

Figura 3. Evolución del precio por gigabyte de almacén en computadores personales

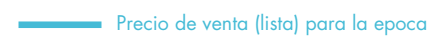

Precio de venta ajustado por inflación en EEUU a 2016

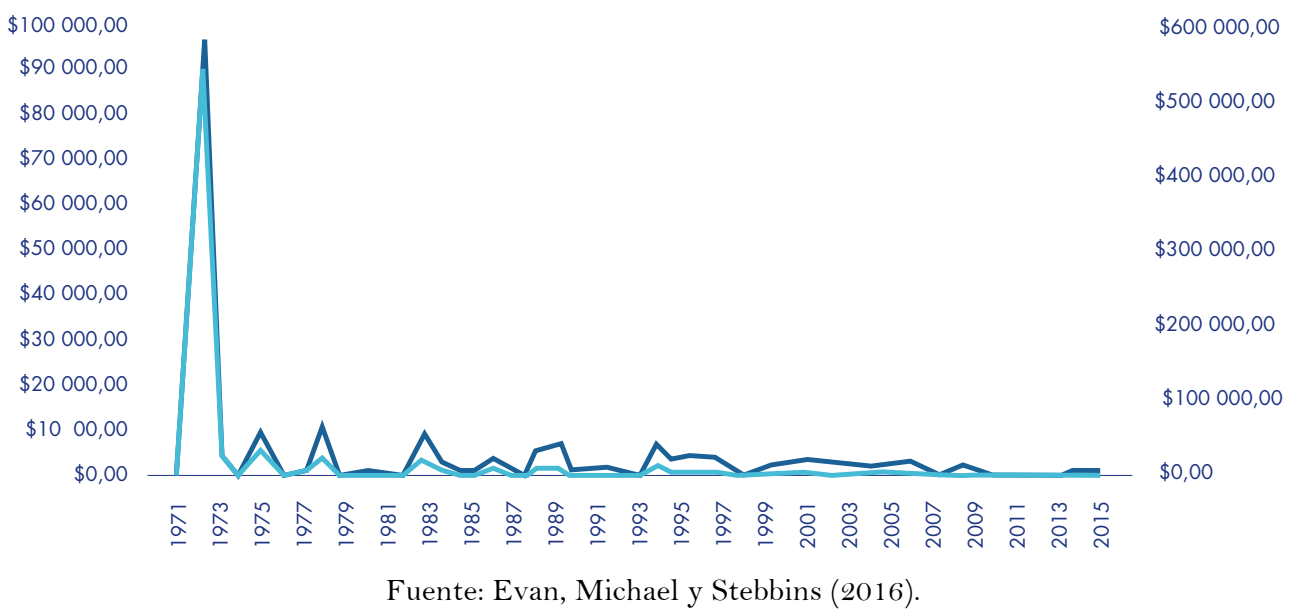

Entre los finales de los años setenta y el siglo pasado, las computadoras personales comenzaron a proporcionar mejoras en los atributos gráficos, dispositivos de almacenamiento e incremento substancial en la capacidad de procesamiento hasta llegar a lo que conocemos hoy en día. En la Figura 4, se observa la tendencia histórica de cómo han venido declinando los precios de almacenaje en estos dispositivos. Sin embargo, está época se caracterizó por la forma en que se procesaban los datos, realizaban los negocios y se interactuaba a nivel global.

Figura 4. Tendencia histórica del precio por gigabyte de almacenaje

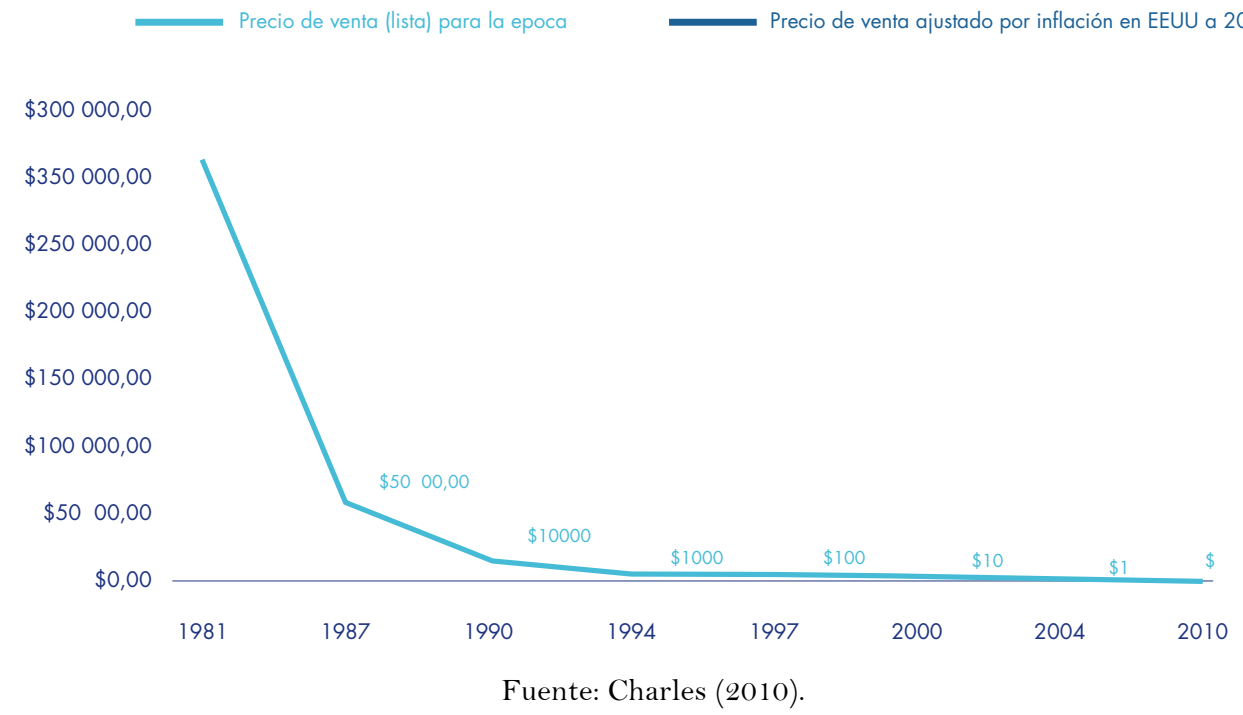


A medida que las computadoras personales iban evolucionando la percepción de los individuos comienza a cambiar, ya que surgen una serie de dispositivos que serían el reemplazo de los computadores personales: entre ellas se destacan el Apple II en 1977, el Commodore VIC-20 en 1980 y el PC de IBM en 1981, los cuales fueron los más populares para su época al momento de su lanzamiento. El Commodore 64 vendió cerca de 20 millones de unidades para convertirse en el ordenador personal con mayores ventas de todos los tiempos (Lean, 2016).

Con el inicio de los años 90, se dio una masificación en el uso del Internet, por lo cual, los computadores debían ser construidos para ser fáciles en su uso y acceso a nivel de precio. Ejemplo de esto fue el iMac en 1998, que, gracias a su interfaz gráfica, uso de un navegador de red fácil de manejar y la incorporación del mouse facilito el acceso la red a miles de familias estadounidenses. Por consiguiente, al pasar los años el número de unidades aumentó y la creciente calidad de los componentes permitió a los fabricantes elaborar dispositivos más pequeños, más baratos y ligeros que podrían hacer mucho más.

En 2005, los ordenadores portátiles se vendieron por primera vez y empiezan a desplazar a las PC en los Estados Unidos de Norteamérica. La llegada del iPhone en 2007 marcó el comienzo de una era en la que la informática se convirtió en verdaderamente móvil. Otros dispositivos de mano como el iPad ofrecen otra opción. En 2015, se vendieron más tabletas que todas las computadoras portátiles y de escritorio combinadas por primera vez (Rapaport y Ricadela, 2013).

Es por este motivo que el dominio en el uso de las TIC y las capacidades de adopción en el uso de las tecnologías innovadores pasan a jugar un papel por parte del teletrabajador y es por ellos que se considerar parte de las variables ser consideras dentro del estudio para evaluar su contribución con el impulso de la productividad y competitividad empresarial.

\section{La transición de lo físico a lo virtual}

El inicio del siglo XXI, se ha caracterizado por un fenómeno muy particular, la transición de las labores de tradicionales del mundo físico al mundo virtual o digital. El mundo físico está caracterizado por objetos tangibles, finitos y temporales, con una vida útil restringida y elaborados con la finalidad de responder una necesidad puntual (Drucker, 1993), mientras que el mundo virtual o digital está caracterizado por la no obsolescencia, la permanencia infinita, su intangibilidad y con la respuesta a múltiples necesidades de los individuos, que han venido ganando un terreno importante dentro de nuestra sociedad, llevándonos al mundo de la era digital o sociedad de la información o conocimiento.

Duque y Uribe (2015), en sus hallazgos mencionaron que la tecnología ha causado un gran impacto en la forma que se crea, se comparte y se almacena la información, convirtiéndola en un recurso ilimitado, donde los individuos pueden tener acceso a ella. Una consecuencia de este fenómeno, es que otras variables pasan a tener especial importancia como son: 1) El espacio, al ser los datos de acceso ilimitado, las personas comienzan a preocuparse por el espacio disponible y requerido para poder almacenarla; 2) El medio ambiente, caracterizado por la necesidad de recolección creciente de datos que demanda de forma creciente de la tecnología, la fabricación de dispositivos con una mayor capacidad de almacenamiento, ocasionando un mayor uso de recursos naturales en su construcción y una aparición de periodos de obsolescencia de la tecnología cada vez más cortos (Duque y Uribe, 2015).

El Internet es en la actualidad una de las principales herramientas usadas para la búsqueda, uso y recopilación de información, permitiendo que millones de personas puedan tener acceso a la misma con solo dar un clic en sus computadoras, sin importar la geografía, zona horaria o idioma, ya que con el pasar de los tiempos surgen instrumentos tecnológicos que acceden a los usuarios sortear todo este tipo de obstáculos y permite el acceso de forma amigable, fácil y sencilla para su manipulación. Estás tecnologías se basan en la indagación en función de palabras clave, que minimizan el tiempo de procesamiento y facilitan la ejecución de cualquier tarea, es por este motivo que el uso de las operaciones vía Internet por parte del teletrabajador puede generar una contribución en el logro de las productividad y competitividad empresarial, siendo esta otra variable a ser considerada dentro del estudio propuesto.

Otro elemento importante a ser considerado es la contribución a la disminución de la huella de carbono ya que, al estar la información disponible en un sitio central, disminuye los procesos de duplicación o copia, así como el desperdicio de papel y la disminución substancial del espacio físico (CAIT, 2015).

A nivel de los sectores gubernamentales, académicos y empresariales existe un gran impulso para garantizar la perpetuidad de la información y su medio de acceso, por lo cual el almacenamiento digital se ha convertido en una prioridad para todos estos sectores, permitiendo el acceso a la consulta una y otra vez de manera infinita y más 
importante aún, de manera digital donde la accesibilidad se vuelve a su vez infinita e ilimitada (eLAC, 2015).

\section{El impacto en los factores de producción}

En muchas estadísticas la productividad se mide como "valor agregado", es decir la cantidad de producción total, menos ciertos recursos empleados para producir como producción en proceso, materias primas, energía, servicios externos comprados, y otros recursos de entrada.

Existen muy pocas estadísticas e indicadores actualizados sobre productividad en América Latina y el Caribe, sin embargo, en este trabajo se ha recabado información útil que muestra tendencias y el comportamiento de algunos de los factores de la productividad (trabajo, capital, gestión empresarial, recursos).

Existe información de la Organización Industrial del Trabajo (OIT, 2008) sobre productividad en el trabajo (uno de los factores de productividad): También se cuenta con cierta información de la Comisión Económica para América Latina y el Caribe (CEPAL, 2008), el Banco Interamericano de Desarrollo (BID, 2001), la Corporación Andina de Fomento (CAF, 2006) y la Organización para la Cooperación y Desarrollo Económico (OECD, 2008) en donde se evidencia lo siguiente:

Considerando el crecimiento demográfico, el ingreso promedio de los latinoamericanos aumentó tan solo 1,5\% anual durante la última década, por debajo no solo de varias regiones del mundo en desarrollo, sino también de los países más maduros demográficamente, como son los del mundo desarrollado y los de Europa Oriental. Si la comparación se circunscribe exclusivamente a la población laboral, el ingreso promedio generado por cada trabajador creció en América Latina a una tasa de tan solo 0,7\% anual. Únicamente los países del Oriente Medio y de África tuvieron crecimientos más modestos de la productividad media del trabajo. En las demás regiones del mundo los aumentos fueron superiores a 1,7\% anual y tanto en los países del Sudeste Asiático como en el resto de Asia la productividad media del trabajo creció cerca de $3 \%$ anual.

En la década de los años noventa los indicadores de crecimiento y productividad de América Latina aumentaron dos puntos porcentuales con respecto a la década anterior. Ninguna otra región del mundo experimentó un avance semejante, sin duda en parte porque fue fácil superar la caída de la "década perdida" (los años ochenta). No obstante, aun antes de la "década perdida" el desempeño de América Latina tampoco era comparativamente destacado. De hecho, durante la segunda mitad del siglo XX, a excepción de África, todas las regiones del mundo sobrepasaron a América Latina en crecimiento per cápita. Como resultado, el ingreso per cápita promedio de América Latina pasó del segundo puesto en los años cincuenta, superada solamente por los países desarrollados, a ocupar en los años noventa el quinto puesto entre siete regiones, por encima únicamente de los países pobres de Asia y África.

América Latina y el Caribe se caracterizan por una baja acumulación de capital físico y una muy baja productividad total de los factores. Este escaso dinamismo se agudiza a partir de la década de 1980. En seis países de la región, entre los que se incluyen las economías de mayor tamaño, la tasa de crecimiento anual de la productividad total de los factores exhibió una tendencia decreciente en los años setenta y fue negativa durante la crisis de la deuda externa y la denominada crisis asiática. En promedio, en todo el período analizado, el crecimiento de la productividad total de los factores se mantuvo en niveles muy bajos.

Sin embargo, la evolución tecnológica ha traído consigo la innovación en los procesos de manufactura de las nuevas tecnologías, lo cual ha generado una reducción en los costos laborales, procesamiento de la información y costos de capital (Rischel y Burns, 1997); es decir, las nuevas tecnologías están influyendo de forma favorable en los factores de producción, ocasionando una mejora en la productividad total (produciendo más con menos). En Latino América, el incremento de la productividad se ha dado por la transferencia y ajuste de esta ciencia, lo cual ha desarolado destrezas y habilidades en los trabajadores locales, que no solo permite replicarla, sino que mejoran la capacidad de producción de las mismas en menor tiempo y costo (De Marco, 2018).

Adicionalmente, las tecnologías de información y comunicaciones proveen mejoras considerables en las velocidades de procesamiento de la información, debido a la mejora de la conectividad. Una consecuencia directa, es la reducción los costos de transacción, por medio de los pagos por internet o en línea. Por otro lado, la capacidad de acceder a información de forma inmediata, conectarse con otros usuarios y clientes, y recopilar información e ideas, ha creado dentro de los factores de producción la importancia de una red. Metcalfe (2013) en su estudio logró realizar la conceptualización del valor de una red como la relación proporcional existente entre los usuarios conectados y las posibilidades de creación de valor económico y social por medio del uso de las redes. 
La evolución de las TIC se logró gracias a la mejora y miniaturización de los circuitos integrados contenidos en cada uno de los dispositivos, esto gracias a la ley de Moore, que planteaba que el número de transistores que se pueden incorporar dentro de un circuito integrado sin mayores costos de producción se puede duplicar cada dieciocho meses (Kaldasch, 2014). Esto ha permitido que durante décadas los costos de producción hayan venido en descenso, por consecuencia los dispositivos electrónicos en la actualidad son más económicos, veloces y potentes que sus versiones previas. Spence (2012), como parte de su hallazgo mencionaba que el incremento en la productividad comenzó a detectarse cuando se dio inicio a la conectividad a Internet, en donde lo primordial no era la capacidad de computo, sino la pertenencia de la red de redes, a partir de este punto comienza a tener un papel primordial el fenómeno de la externalidad de la red.

Adicionalmente, el impacto económico de las redes basadas en los computadores personales se ha desarrollado a través de tres tendencias de forma paralela:

1. La automatización de la información y la capacidad de gestionar los datos, ha permitido eliminar costos y errores laborales, aumentando la velocidad de procesamientos y la productividad; es decir, la evolución tecnológica reduce el costo laboral y elimina el costo de transacción. Como consecuencias las empresas se han visto obligadas a reconfigurar sus estructuras organizativas a nivel global, ampliando el rango de transacciones gestionables y promoviendo nuevos mecanismos de innovación dentro de la organización a través de la conectividad (Spence, 2012).

2. La migración de numerosas tareas, actividades de adquisición y procesamientos de información hacia sitios en la World Wide Web, ha logrado la reducción en los costos y tiempos de búsqueda de la información y facilitado el hallazgo de ideas y contenidos desconocidos para el individuo. Como consecuencia de esto la producción de contenidos crece más rápidamente que el número de conexiones a la red, siendo los motores de búsqueda de la internet los elementos centrales que permiten facilitar está explosión de oportunidades (Asim y otros, 2017). La rápida y creciente evolución de la World Wide Web, ha permitido que estos motores de búsqueda se conviertan en una herramienta que puede atender una gama de sectores de la sociedad de forma diversa (Gobierno, Universidades, Cámaras Empresariales, Firmas Consultores, Empresas e Individuos). Estos buscadores a su vez se han convertido en habilitadores de nuevas tecnologías disruptivas, en parte debido a su versatilidad y modelo de negocio usado, ya que realizar la interconexión entre la persona y la necesidad complementaria requerida, como es el caso de las tecnologías como Amazon, EBay, Alibaba, Google, Facebook, Skype y Twitter. En el siglo XXI, la combinación de los diferentes dispositivos electrónicos disponibles y los servicios de computación en la nube, reducen de forma drástica el uso de computadores portátiles, el uso de papel, el uso de llamadas telefónicas y la disminución de los traslados a la oficina, entre otras cosas.

3. El acceso de forma inmediata a recursos humanos valiosos para cada uno de los individuos (familiares, colegas, socios, entre otros) sin importar la localización geográfica en donde se encuentren, con posibilidad de usarlos en el procesamiento y cadenas productivas en los mercados dentro de una economía global. Accediendo al mejor talento humando a nivel global, gracias a la capacidad de conexión que ofrece el Internet, dando acceso a personas calificadas para que realicen trabajos de forma remota, debido a la carencia de movilidad del individuo a su centro laboral tradicional (Deshpande, 2018).

Spence (2012), menciona que en el caso de la primera tendencia los efectos de mejora pueden ser medibles, pero en el caso de la segunda y tercera tendencia todavía no existen en la actualidad herramientas que permitan realizar está medición.

Harris (2008), desde su perspectiva de la evolución de la conectividad y el acceso a la información, en donde se muestra que el individuo moderno está basado más en decisiones regidas por factores externos que internos, es decir, mucha de la información que rige la toma de decisiones de los seres humanos no se encuentra basado en los genes o en la memoria, sino que se encuentra almacenada en bibliotecas o instituciones. El surgimiento a lo largo de la historia de la escritura hasta las bases de datos informáticas y la inteligencia artificial, han permitido lograr un ahorro de tiempo en los procesos de redescubrimiento, facilitando la reproducción y diseminación del conocimiento, actividades y experimentos.

Las TIC han permitido poder almacenar la historia y cultura del ser humano desde sus orígenes hasta la actualidad (Diaz y Darío, 2013). La siguiente etapa de la evolución tecnológica será la capacidad de procesamiento, medida por la conectividad y el ancho de banda de las comunicaciones. En la academia Flynn (2007), mencionan que el uso de las 
TIC han permitido al ser humano tomas decisiones de forma menos compleja debido a la simplificación y desglose de los problemas, como resultado de la mejora en los procesos educativos, sin que el cerebro haya cambiado de estructura o tamaño de forma substancial, esto debido a la ayuda que ofrece la conectividad en la evolución central y las capacidades de los individuos, este fenómeno también es conocido en la literatura como el "efecto flynn".

En la última década, la telefonía móvil o celular ha sido un elemento clave para poder brindar conectividad a muchos individuos, en especial en los países latinoamericanos, ya que ha brindado acceso a las zonas rurales al Internet y las comunicaciones digitales; por lo cual el reto de proveer el acceso a todos los individuos a nivel mundial se ve superado, quedando como nuevo reto la profundización en el uso de aplicaciones de alto valor agregado, en donde se requiere una constante ampliación e innovación tanto de las redes de comunicación fijas, como móviles para poder responder al nivel de demanda cada uno de los países.

Es por este motivo que si el teletrabajador logra fomentar su manejo del tiempo y autonomía por medio del apoyo de la tecnología podrá contribuir con la productividad y competitividad empresarial, es por ello por lo que se considerar estas dos variables como parte del estudio.

\section{Objetivo}

El objetivo del estudio consistió en identificar los factores clave que pueden incidir de forma positiva en la productividad laboral a través del teletrabajo

\section{Metodología}

El tipo de investigación cualitativa y el alcance del estudio fue descriptivo y exploratorio. Es descriptiva, ya que su finalidad consistió en explicar los factores claves que impulsan a los teletrabajadores profesionales a ser más productivos y competitivos, entiendo el nivel profesional como el valor definido en la escala de CINE de la UNESCO.

En el Cuadro 1 se presentan los niveles de la escala de CINE, en donde a partir del nivel 5 es considerado el nivel profesional, debido a que es el nivel donde se considera que las actividades laborales son realizadas por profesionales con estudios de nivel superior (licenciaturas, ingenierías, entre otros).

Cuadro 1. Clasificación internacional normalizada para la educación (CINE)

\begin{tabular}{|c|c|}
\hline Nivel & Definición \\
\hline $\begin{array}{c}\text { Nivel } 0 \\
\text { (Educación preprimaria) }\end{array}$ & $\begin{array}{l}\text { Brinda ayuda y soporte oportuno en el desarrollo social, físico, } \\
\text { cognitivo y emocional de los niños e infantes. Ejecuta la primera } \\
\text { inducción formal y organizada fuera del hogar. }\end{array}$ \\
\hline $\begin{array}{l}\text { Nivel } 1 \\
\text { (Educación primaria) }\end{array}$ & $\begin{array}{c}\text { Orientada a una educación formal del primer ciclo de la } \\
\text { educación básica. }\end{array}$ \\
\hline $\begin{array}{c}\text { Nivel } 2 \\
\text { (Primer ciclo de educación secundaria) }\end{array}$ & $\begin{array}{c}\text { Orientada a una educación formal del segundo ciclo de la } \\
\text { educación básica (secundaria baja). Requiere de la aprobación } \\
\text { del nivel } 1 \text {. }\end{array}$ \\
\hline $\begin{array}{c}\text { Nivel } 3 \\
\text { (Segundo ciclo de educación secundaria) }\end{array}$ & $\begin{array}{c}\text { Orientada a una educación formal del ciclo de la educación } \\
\text { secundaria (secundaria alta). Requiere de la aprobación del } \\
\text { nivel } 2 \text {. }\end{array}$ \\
\hline $\begin{array}{l}\text { Nivel } 4 \\
\text { (Educación postsecundaria no terciaria) }\end{array}$ & $\begin{array}{l}\text { Orientada a reforzar los conocimientos adquiridos en la } \\
\text { secundaria con la finalidad de preparar al individuo para el } \\
\text { mercado laboral. Las habilidades, conocimientos y competencias } \\
\text { obtenidos en este nivel se encuentran en una etapa previa de la } \\
\text { educación terciaria, a su vez amplían el conocimiento más que } \\
\text { su profundización, generalmente está orientada a individuos que } \\
\text { han completado el nivel } 3 \text {, bien sea con las calificaciones que } \\
\text { estos requieren para pasar a la educación terciaria o para acceso } \\
\text { mercado laboral (profesional) cuando la conclusión exitosa del } \\
\text { nivel } 3 \text { no brinda este acceso. Requiere la aprobación del nivel } 3 \text {. }\end{array}$ \\
\hline
\end{tabular}


Nivel 5

(Primer ciclo de educación terciaria)
Se caracteriza por un proceso de formación formal de al menos 2 años de duración a tiempo completo, posee una base práctica, orientación hacia ocupaciones específicas y preparación del individuo para el mercado laboral, permiten abrir el camino hacia otros programas de educación terciaria. Incluyen programas académicos debajo de grado en educación terciaria (o equivalente). Requieren la aprobación del nivel 3 ○ 4, dependiendo del nivel de complejidad de los contenidos académicos y formación de niveles anteriores.

Es una educación formal que permite la obtención de un título en un periodo de tres años en jornada completa y llegar hasta cuatro años en programas de mayor duración, brinda al individuo habilidades, conocimientos y competencias profesional - académicas de base teórica o práctica. Frecuentemente son impartidas por instituciones de educaciones como universidades o institutos profesionales. Los programas no requieren necesariamente desarrollar una tesis o proyecto de graduación.

Requieren la aprobación del nivel 3 o 4 y habilitan al individuo para continuar estudiando en el nivel 7.

Se enfoque en proporcionar a las personas habilidades, conocimientos y competencias profesionales y académicas con un nivel avanzado. Pueden comprender el uso de componentes de investigación y el desarrollo de una tesis, comprenden los programas de primer título, cuya duración es de cinco años a tiempo completo, considera estudios profesionales especializados como ingeniería, medicina, entre otros, que no requieren del desarrollo de una tesis.

Su duración comprende a tres años de estudio a tiempo completo y comprende a realización de estudios avanzados e investigaciones de carácter original, requieren el desarrollo de una tesis que provea un aporte al conocimiento, adicionalmente puede ser publicable, son impartidas por instituciones de educación superior (universidades) dedicadas a la investigación.

Fuente: UNESCO, 2013.

Es exploratoria, debido a que se desea conocer la relación existente entre el teletrabajador y la empresa, y como las tecnologías contribuyen con esta relación para alcanzar las metas de productividad y posición competitiva desea por la organización.

Hernández, Fernández y Baptista(2016) establecieron que los diseños no experimentales se clasifican en longitudinales y transversales, en donde el transversal se caracteriza por realizarse en un único momento, siendo este un atributo de este estudio, ya que toma como componente la situación actual de las empresas que están realizando sus procesos de selección, evaluación y certificación de trabajadores a nivel profesional de forma remota en la República de Costa Rica para el 2017.

Por consiguiente, la temporalidad del estudio fue transversal. Glasser y Strauss (1967) indicaron que toda investigación en la cual se descubran hechos, datos y acciones a partir de la comprensión generalista de diferentes exploraciones comparativas es considerada con un enfoque de construcción de una teoría, siendo este otro atributo del estudio realizado.

Para Schettini y Cortazo (2015), la investigación cualitativa utiliza en el transcurso del abordaje de la investigación el análisis profundo, pero además el histórico, el contextual, lo cual contribuye a ir circunscribiendo el campo particular de la investigación, cualitativa.

Por último, Creswell (2014) mencionaba que el enfoque cualitativo son aquellos que se caracterizan por apoyarse en los estudios de teorías, análisis de casos , la fenomenología, además se apoya en métodos emergentes, preguntas semi abiertas, observación de datos, entrevistas, audio y video, también análisis de texto e imágenes, así como patrones de interpretación; esta es una investigación por lo tanto cualitativa y en función de los resultados obtenidos se construye un instrumento (encuesta) con las variables identificadas. Luego, a través de una investigación cuantitativa se valida su correlación en función de una variable independiente.

En el plano instrumental comprendió el uso de mecanismos que permitieron la recolección de datos categóricos, ordinales y nominales como parte del análisis cualitativo. McMillan y Schumacher (2005) indicaron que las 
investigaciones cualitativas usan estas técnicas para recoger un conjunto de datos que son perfeccionados a medida que avanza en el desarrollo del estudio, por medio de la adquisición de más datos como mecanismo de validez y confiablidad. Por consiguiente, para alcanzar este objetivo se realizó una entrevista en profundidad de tipo no estructurada a siete representantes sectoriales de la sociedad que realizan teletrabajo de forma exitosa en sus organizaciones y están alcanzando mejoras en la productividad laboral.

La población del estudio estuvo constituida por cinco empresas activas y en operación durante el año de 2017 ubicadas en la República de Costa Rica, que realizaron actividades laborales bajo la modalidad del teletrabajo a nivel profesional y obtuvieron en sus resultados el logro de las metas establecidas a través de los rendimientos alcanzados por sus teletrabajadores.

Por consiguiente, con la finalidad de obtener preguntas dirigidas, información importante, significativa y relevante del objeto de estudio, acontecimientos y dimensiones objetivas y subjetivas como leyendas, mitos, pensamientos, creencias y valores para lograr el entendimiento desde una visión global (Buendía, Colás y Pilar, 1998) se seleccionó como instrumento de la investigación a la entrevista.

Ruiz (2003) mencionaba que la entrevista puede, a su vez, ser catalogada en profundidad, caracterizándose principalmente por: 1) ser Individual, ya que establece una conversación personalizada entre el entrevistador y el entrevistado; 2) ser holística, ya que extrae los significados de la persona entrevistada y construye un entorno en función del tema en estudio y 3 ) ser de carácter no directivo, dando a comprender que la entrevista es de tipo no estructurada, siendo estos tres atributos los utilizados en el estudio.

Con los resultados, se aplicó la técnica de conteo de palabras que permitió el agrupamiento de elementos basados en la similitud de la información proporcionada por los entrevistados, a través, de palabras repetidas contenidas dentro de los ejes principales de los factores que determinan la relación ganar-ganar entre el trabajador y la empresa por medio del teletrabajo.

\section{Discusión y resultados}

Los resultados obtenidos a partir de la aplicación de los instrumentos tenían como finalidad el poder identificar los factores clave para el teletrabajo que pudiesen impactar de forma positiva en la productividad y competitividad empresarial.

Por lo tanto, una vez culminada cada una de las entrevistas en profundidad y transcriptas a documentos en formato Microsoft Word se realizó al análisis del texto de forma electrónica a través funcionalidad de consulta de frecuencia de palabras contenida dentro de la aplicación de NVivo Pro (versión 11), con el objetivo de identificar las características, tales como palabras repetidas y frases repetidas de forma general que permiten dar origen a la codificación de categorías como factores dentro una matriz de resumen en función de la información recopilada. Por consiguiente, los resultados identificados fueron los siguientes:

\section{a. Dominio en el uso de las TIC}

Las empresas entrevistadass coinciden con un $54,55 \%$ de las respuestas que esta variable es el factor mas importante para alcanzar las metas de productividad y competitividad empresarial. A su vez expresan que para que esta variable pueda alcanzar su objetivo se requiere que el teletrabajador posea el dominio y uso de las TIC, $50 \%$ de las empresas entrevistas coinciden con este resultado. Es por ellos que ambas variables se identifican con una posible correlacion, pero no se logra comprobar debido al tamaño de la muestra seleccionada.

Gibbons, Limoges, Nowotny, Schwartzman, Scott y Trow (1997) mencionaron que desde la aparición de la revolución científico técnica que transcurre en la contemporaneidad y de los extraordinarios avances tecnocientíficos que le son consustanciales, se está asistiendo a cambios fundamentales en la forma de producir el conocimiento científico, tecnológico y cultural, siendo este nuevo modo característico de la reflexividad, la heterogeneidad y la existencia de una gama más amplia de criterios para juzgar su naturaleza y calidad.

Sin duda alguna, desde el inicio de la masificación de la red de Internet en 1989 y su constante evolución, ha permitido que las organizaciones cambien en su forma de trabajar y por ende sus trabajadores, convirtiéndose en un requisito para la actividad laboral el uso y dominio de las tecnologías de información y comunicaciones por parte de los trabajadores profesionales dentro de las organizaciones. Sin embargo, como se evidencia en los resultados es un esfuerzo en conjunto, ya que el sector empresarial requiere del apoyo del sector educación en la formación de los profesionales calificados en el uso de estas tecnologías. 


\section{b. Manejo del tiempo}

Las empresas indicaron con un $36,36 \%$ de las respuestas que esta variable es el segundo factor mas importante y posee una posible relacion con el dominio en el uso de las TIC y la autonomia, ambas variables coindicen con un $30 \%$ de las respuestas obtenidas.

La gestión del tiempo es un elemento importante para las empresas, en especial al momento de realizar actividades profesionales bajo la modalidad del teletrabajo. Griffiths (2003) en su investigación abordó el problema de la gestión del tiempo, desde la perspectiva de cómo aplicar los procesos por parte del teletrabajador para poder autorregular el uso eficiente del dominio tiempo y tomando como base la teoría de la autorregulación, modifica la escala del comportamiento de la gestión del tiempo (TMBS) para adecuarla al teletrabajo, en donde incluye los siguientes factores hipotéticos: fijación de objetivos, mecánica del tiempo, estrategias cognitivas, autorecompensa, autocastigo y control percibido de hora. Los resultados obtenidos fueron que todos los factores, excepto el control percibido del tiempo, estaban asociados con el orden superior construcción de gestión del tiempo; es decir, desde la perspectiva empresarial, ya existen estudios en la academia que pueden contribuir con el logro de la productividad del teletrabajador.

c. Autonomía

En esta variable las empresas coincidieron en $27 \%$ posicionando en el tercer nivel de importancia y con una aparecente relacion con el dominio en el uso de las TIC y manejo del tiempo con un $30 \%$.

Gajendran y Harrison (2007) en su investigación indicaron que las actividades laborales basadas en la modalidad del teletrabajo requieren de un alto nivel de autonomía por parte del teletrabajador en la planeación y ejecución de sus actividades laborales con la finalidad de alcanzar las metas establecidas, en donde la organización debe ser flexible, ya que si se priva de la autonomía y la capacidad de decisión, se elimina el componente autoformativo y la toma de decisiones importantes por parte del trabajador.

\section{d. Operaciones en vía internet}

En la cuarta posicion se ubica esta variable con 26,27 \%. En el 2017, 46,8 \% de la población mundial accedió a Internet y para el 2021 se estima que el crecimiento sera a 53,7 \% de la población mundial, lo cual da evidencia de un crecimiento acelerado en los productos y servicios digitales, para lo cual las empresas se ven en la necesidad de incursionar cada vez más en el desarrollo de sus actividades a través de la Internet (Clements, 2019).

\section{e. Adopción en el uso de TIC innovadoras}

Por último, se destaca con un $20 \%$ esta variable entre las empresas entrevistadas. Schumpeter en 1954, como resultado de sus estudios, identificó que la innovación es una dimensión crítica del cambio económico, siendo el uso de las tecnologías innovadores uno de los instrumentos claves para lograrlo (Pol y Carroll, 2006). Por su lado, Porter (1990), mencionaba que la competitividad de las organizaciones depende de la capacidad de innovar que tenga; es decir, la empresa alcanzará una ventaja una ventaja competitiva mediante las innovaciones, siendo las tecnologías el instrumento clave para el logro de esto.

Es importante destacar que debido a que el análisis realizado es bajo un enfoque cualitativo, no es posible determinar el tipo de impacto que produce cada uno de los factores, su fuerza, magnitud y dirección, ya que se requiere la realización de un estudio cuantitativo de carácter no lineal, el cual no fue abarcado como parte de la investigación propuesta.

\section{CONCLUSIONES}

El teletrabajo ha sido posible a que los costos de los hardware, los softwares, la velocidad del Internet, el almacenamiento de datos, así como el procesamiento de datos, han bajo significativamente, así como el desarrollo de nuevas aplicaciones y las grandes cantidades de horas que se debe invertir en el desplazamiento los trabajadores con ello en detrimento de su calidad de vida, ha propiciado al teletrabajo con ello se contribuye a disminuir también la contaminación por el uso de transporte basado en petróleo; han impulsado el moderno tipo de trabajo, el trabajo a distancia o teletrabajo.

Además, surgen cambios en los paradigmas de la vida del ser humano, no solo en lo laboral sino en lo cotidiano. El proceso de innovación acelerado y la aparición de nuevas tecnologías que faciliten el desarrollo de las actividades laborales del individuo, hacen que las empresas las tomen en consideración como un factor estratégico para mejorar su ventaja competitiva, como a su vez impulsar el crecimiento económico, generación y formación de trabajo. 
Aghion, Bloom, Blundell, Griffith y Howitt (2005), como parte de su investigación, plantearon que las empresas que se encuentran en entornos muy competitivos se ven en la necesidad de innovar, en donde la distancia tecnológica promedio entre líderes y seguidores aumenta con la competencia.

El teletrabajo ha permitido deslocalizar el trabajo in situ tanto de manera local como global, ahora el teletrabajador, pude estar en cualquier parte del mundo y cumplir con sus objetivos de trabajo.

También, los procesos de innovación en la búsqueda de nuevas tecnologías se convierten en la motivación en el sector empresarial, tomando en consideración los criterios de penetración hacia mayores usuarios, como es el caso de Internet, donde en la década de los años 90 se medía su uso por cantidad de usuarios conectados a la Internet y en el año 2015, se mide como cantidad de conexiones por usuario a la internet. Otro criterio a tomar en consideración es el precio de los dispositivos; debido a la disminución de los ciclos de vida de productos, se presenta un rápido fenómeno de obsolescencia en los dispositivos electrónicos y cibernéticos, lo cual causa que los fabricantes busquen nuevos mecanismos para colocar precios competitivos y masificar la venta de sus productos con los lanzamientos comerciales de los mismos a través de estrategias; por otro lado los productos que entran en proceso de obsolescencia se vuelven atractivos a otros nichos de mercado de los consumidores.

El análisis cualitativo desprendió que las empresas que ya están realizando actividades profesionales en función del teletrabajo, consideran que la adopción en el uso de las TIC innovadoras es el elemento más importante, esto debido a que actualmente realizan sus actividades profesionales bajo esta modalidad, por lo cual sus objetivos se enfocan en la mejora continua para incrementar los rendimientos marginales alcanzados por los teletrabajadores en sus metas planteadas. No obstante, este criterio no necesariamente es el más importante para las empresas que no han incursionado en esta modalidad laboral y que no pudo ser determinado por la carencia de conocimiento y experticia en la relación entre teletrabajo profesional y estrategia empresarial.

Los diferentes grupos de interés evidencian que los factores requeridos en el sector empresarial para el desarrollo de las competencias laborales para el teletrabajo están basados en el desarrollo de destrezas y habilidades del teletrabajador por medio del uso de herramientas tecnológicas para así lograr alcanzar las metas establecidas como parte del puesto de trabajo. Bueno y Morcillo (1997) mencionaron que la unión entre las competencias laborales y la estrategia generan una distinción en la organización, ya que logran alcanzar rendimiento superiores por parte de los trabajadores, lo cual da evidencia de un posible impacto en la productividad de los teletrabajadores y la obtenición de una ventaja competitiva por parte de la organización, siendo este el caso de las empresas entrevistadas.

A su vez, la participación de los diferentes actores de la sociedad permitió identificar por medio de un consenso sectorial el nivel de importancia que tiene para las empresas cada uno de los factores identificados. Preston y O’Bannon (1997) mencionaron que cuando diversos grupos de interés participan y suplen sus necesidades, trae como consecuencia el aporte de conocimiento, tiempo para atender y coordinar actividades, lo cual fue un elemento muy importante durante el desarrollo del trabajo de investigación debido a la carencia de trabajos de investigación en la literatura en referencia a competencias laborales para el teletrabajo, permitiendo así desarrollar el estudio desde un enfoque aplicado.

De la investigación, se puede inferir que el teletrabajo se ha impulsado basado principalmente en cinco factores, los cuales son:

\section{a. Dominio en el uso de las TIC}

El $54,55 \%$ de las respuestas, dice que esta variable es el factor más importante para mejorar la productividad y competitividad empresarial. Siendo que se requiere que el teletrabajador posea el dominio y uso de las TIC, $50 \%$ de las empresas entrevistas coinciden con este resultado.

Gibbons, Limoges, Nowotny, Schwartzman, Scott y Trow (1997) mencionaron que desde la aparición de la revolución científico técnica que transcurre en la contemporaneidad y de los extraordinarios avances tecnocientíficos que le son consustanciales, se está asistiendo a cambios fundamentales en la forma de producir el conocimiento científico, tecnológico y cultural, siendo este nuevo modo característico de la reflexividad, la heterogeneidad y la existencia de una gama más amplia de criterios para juzgar su naturaleza y calidad.

\section{b. Manejo del tiempo}

El 36,36\% de las respuestas de esta variable es el segundo factor más importante y posee una posible relación con el dominio en el uso de las TIC y la autonomía, ambas variables coindicen con un 30\% de las respuestas obtenidas. 
La gestión del tiempo es un elemento importante para las empresas, en especial al momento de realizar actividades profesionales bajo la modalidad del teletrabajo. Griffiths (2003) en su investigación abordó el problema de la gestión del tiempo, desde la perspectiva de cómo aplicar los procesos por parte del teletrabajador para poder autorregular el uso eficiente del dominio tiempo y tomando como base la teoría de la autorregulación, modifica la escala del comportamiento de la gestión del tiempo (TMBS) para adecuarla al teletrabajo.

\section{c. Autonomía}

En esta variable las empresas coincidieron en $27 \%$ posicionando en el tercer nivel de importancia. Siendo que Gajendran y Harrison (2007) en su investigación indicaron que las actividades laborales basadas en la modalidad del teletrabajo requieren de un alto nivel de autonomía por parte del teletrabajador en la planeación y ejecución de sus actividades laborales con la finalidad de alcanzar las metas establecidas.

\section{d. Operaciones vía internet}

En la cuarta posición se ubica esta variable con $26,27 \%$, para el 2021 se estima que el crecimiento será acelerado en los productos y servicios digitales, para lo cual las empresas se veran en la necesidad de incursionar cada vez más en el desarrollo de sus actividades a través de la Internet (Clements, 2019).

\section{e. Adopción en el uso de TIC innovadoras}

Por último, se destaca la adopción en el uso de TIC innovadoras, con un $20 \%$ entre las empresas entrevistadas. Ya Schumpeter en 1954, identificó que la innovación es una dimensión crítica del cambio económico, siendo el uso de las tecnologías innovadores uno de los instrumentos claves para lograrlo (Pol y Carroll, 2006). Por su lado, Porter (1990), mencionaba que la competitividad de las organizaciones depende de la capacidad de innovar que tenga; es decir, la empresa alcanzará una ventaja una ventaja competitiva mediante las innovaciones, siendo las tecnologías el instrumento clave para el logro de esto.

Por lo cual se puede inferir que los factores claves para que se dé el teletrabajo son estos cinco factores a saber: el uso y dominio de las tecnologías de la información y comunicación, la administración del tiempo, la autonomía, el uso intensivo de Internet y la innovación en las tecnologías disruptivas.

Es importante destacar que debido a que el análisis realizado es bajo un enfoque cualitativo, no es posible determinar el tipo de impacto que produce cada uno de los factores, su fuerza, magnitud y dirección, ya que se requiere la realización de un estudio cuantitativo de carácter no lineal, el cual no fue abarcado como parte de la investigación propuesta

\section{REFERENCIAS}

Aghion, P., Bloom, N., Blundell, R., Griffith, R. y Howitt, P. (2005). Competition and innovation: An inverted-U relationship. The Quarterly Journal of Economics, 120(2), 701-728.

Asim, S., Nazri, M., Norhamreeza, A., Sundas, K., Muhammad, A., Arif, U. y Salfarina, A. (2017). The Impact of Search Engine Optimization on The Visibility of Research Paper and Citations. International Journal of Informatics visualization.

BID (2001). Crecimiento, productividad y Competitividad en América Latina.

Bueno, E. y Morcillo, P. (1997). Dirección estratégica por competencias básicas distintivas: Propuesta de un modelo: Instituto Universitario de Administración de Empresas.

CAF (2006). Ruta para la transformación: diversificación con productividad.

CAIT (2015). Climate Analysis Indicators Tool: WRI’s Climate Data Explorer. Washington, DC. Recuperado desde: http://cait.wri.org/

Castells, M. (1997). The information age: Economy, society and culture. Vol. 2, The power of identity: Blackwell.

CEPAL (2008). La transformacion productiva, 20 años despues.

Charles G., K. (2010). The decline and fall of disk storage prices.

Clement, J. (2019). Mobile internet usage worldwide: Statistics \& Facts. Statista. Recuperado desde: https://www. statista.com/topics/779/mobile-internet/

Computer History Museum: Internet History from 1962 to 2015. (2016). 
Computer History Museum: Timeline of Computer History. (2016).

Creswell, J. (2014). Research design-qualitative, quantitative \& mixed methods approaches. (Vol. 4): Sage Publications Inc. De Marco, N. (2018). The Growing Role Of Latin America In The Technology Industry. Forbes Technology Council. Deshpande, A. (2018). Talent Acquisition through Technology. IOSR Journal of Business and Management (IOSR-JBM). Diaz, A. y Dario, W. (2013). Hacia una evolucion historica de las TIC. Hallasgos, vol. 10, Bogota, Colombia.

Drucker, P. (1993). Post-Capitalist Society. New York, U.S.A.: Harper Collins Publishers.

Duque, C. y Uribe, M. (2015). Menos es más: La transición de lo Físico a lo Digital. Colegio de Estudios Superiores de Administración. Bogota, 18.

eLAC (2015). El teletrabajo en América Latina. Recuperado desde: http://www.cepal.org/cgi-bin/getprod.asp?xml=/ elac2015/noticias/paginas/5/40835/P40835.xml\&xsl=/elac2015/tpl/p18f.xsl\&base=/elac2015/tpl/top-bottom.xsl

Evan, C., Michael B., S. y Stebbins, S. (2016). The cost of a computer the year you were born.

Flynn, J. (2007). What is intelligence?: Beyond the Flynn effect: Cambridge University Press.

Gajendran, R. y Harrison, D. (2007). The good, the bad, and the unknown about telecommuting: meta-analysis of psychological mediators and individual consequences: American Psychological Association.

Gibbons, M., Limoges, C., Nowotny, H., Schwartzman, S., Scott, P. y Trow, M. (1997). La nueva producción del conocimiento. La dinámica de la ciencia y la investigación en las sociedades contemporáneas, 20.

Glasser, B. y Strauss, A. (1967). The development of grounded theory. Chicago, IL: Alden.

Griffiths, R. (2003). Time Management in Telework and Other Autonomous Work Environments. Recuperado desde: https://books.google.co.cr/books?id=fTZaNwAACAAJ

Harris, S. (2008). A Million years of evolution: Wetware to Software to Fashionware. Broderick. New York, Atlas \& Co, 42-84.

Hernández, R., Fernández, C. y Baptista, P. (2016). Metodología de la Investigación (Vol. 4a ed.). México: Mc Graw Hill. Hodson, N. (1994). Teleworking \& Employment in Europe. SW 2000 Teleworking Studies. Oxford.

Kaldasch, J. (2014). Evolutionary Model of Moore's Law. Hindawi Publishing Corporation.

Lean, T. (2016). Electronic dreams: How 1980s Britain Learned to love the computer. Bloomsbury Publishing.

McMillan, J. y Schumacher, S. (2005). Investigación educativa una introducción conceptual: Pearson educación.

Metcalfe, B. (2013). Metcalfe's law after 40 years of ethernet. Computer, 46(12), 26-31.

OECD (2008). Compendium of Productivity Indicators.

OIT (2008). Labour productivity and unit labour cost indicators.

Pol, E. y Carroll, P. (2006). An introduction to economics with emphasis on innovation.

Porter, M. (1990). The competitive advantage of nations. Harvard business review, 68(2), 73-93.

Preston, L. y O’Bannon, D. (1997). The corporate social-financial performance relationship: A typology and analysis. Business \& Society, 36(4), 419-429.

Rapaport, L. y Ricadela, A. (2013). Global Tablet Shipments to Overtake PCs by 2015. Recuperado desde: https:// www.bloomberg.com/news/articles/2013-05-28/pc-market-to-decline-7-8-in-2013-as-mobile-devices-gain

Rischel, T. y Burns, O. (1997). The impact of technology on small manufacturing firms. Journal of Small Business Management, Milwaukee, Tomo 35, 2-10.

Ruiz, J. (2003). Técnicas de triangulación y control de calidad en la investigación socioeducativa: Ediciones Mensajero. Bilbao.

Schettini P. y Cortazo I. (2015) Análisis de datos cualitativos en la investigación social : procedimientos y herramientas para la interpretación de información cualitativa, 1ra. edicion, La Plata: Universidad Nacional de La Plata.

Spence, M. (2012). The next convergence: The future of economic growth in a multispeed world: Macmillan.

UNESCO (2013). Clasificación Internacional Normalizada de la Educación (CINE 2011). Recuperado desde: http:// www.uis.unesco.org 